\title{
New Results from NuTeV
}

\author{
V. Radescu ${ }^{1}$ for the $\mathrm{NuTeV}$ Collaboration \\ 1- DESY, Notkestrasse 85, Hamburg D-22607 - Germany
}

\begin{abstract}
The $\mathrm{NuTeV}$ experiment has collected high statistics, high energy samples of $\nu$ and $\bar{\nu}$ charged-current interactions during 1996-1997 using the sign-selected Fermilab neutrino beam. NuTeV has extracted final $\nu$ and $\bar{\nu}$ differential cross sections for DIS single-muon production at $x>0.015$ and average $Q^{2} \sim 15 \mathrm{GeV}^{2}$. Also presented here is the first measurement of $\Lambda_{Q C D}$ from Next-to-Leading Order QCD fits using a theoretical model that fully accounts for heavy quark production.
\end{abstract}

\section{Introduction}

The purely weak nature of neutrino interactions makes them a unique probe of the nucleon structure. In charged-current (CC) neutrino DIS the $\nu(\bar{\nu})$ scatters off a quark in the nucleon via exchange of a virtual $W$-boson. The cross section can be expressed in terms of the structure functions $2 x F_{1}\left(x, Q^{2}\right), F_{2}\left(x, Q^{2}\right)$, and $x F_{3}\left(x, Q^{2}\right)$ :

$$
\frac{d^{2} \sigma^{\nu(\bar{\nu})}}{d x d y}=\frac{G_{F}^{2} M E_{\nu}}{\pi}\left[\left(1-y\left(1+\frac{M x}{2 E_{\nu}}\right)+\frac{y^{2}}{2} \frac{1+\left(\frac{2 M x}{Q}\right)^{2}}{1+R_{L}}\right) F_{2}\left(x, Q^{2}\right) \pm\left(y-\frac{y^{2}}{2}\right) x F_{3}\left(x, Q^{2}\right)\right],
$$

where $G_{F}$ is the Fermi weak coupling constant, $M$ is the proton mass, $E_{\nu}$ is the incident neutrino energy in the lab frame, and $y$, the inelasticity, is the fraction of energy transferred to the hadronic system. $R_{L}\left(x, Q^{2}\right)$ is the ratio of the cross sections for scattering of longitudinally and transversely polarized $\mathrm{W}$-bosons. It relates $F_{2}\left(x, Q^{2}\right)$ and $2 x F_{1}\left(x, Q^{2}\right)$. Relativistic invariant kinematic variables, $x, y$, and $Q^{2}$, can be evaluated in the lab frame using the experimentally measured quantities: $E_{\mu}$, the energy of the outgoing primary charged-lepton, $E_{h a d}$, the energy deposited at the hadronic vertex, and $\theta_{\mu}$, the scattering angle of the primary muon.

$\mathrm{NuTeV}$ is a fixed target deep inelastic neutrino-scattering experiment which took data during 1996-1997 at Fermilab. It combines two important features: the use of a sign selected beam to produce separate $\nu$ and $\bar{\nu}$ beams, and the use of a continuous calibration beam running concurrently with the data-taking, which enables the $\mathrm{NuTeV}$ experiment to considerably improve its knowledge of the energy scale for hadrons and muons to a precision of $0.43 \%$ and $0.7 \%$, respectively [2].

\section{Measurement of the Differential Cross Section}

The differential cross section per nucleon on iron at a given neutrino energy as function of $x, y$ and $E_{\nu}$ is determined from the differential number of events and the flux, $\Phi\left(E_{\nu}\right)$,

$$
\frac{d^{2} \sigma_{i j k}^{\nu(\bar{\nu})}}{d x d y} \propto \frac{1}{\Phi\left(E_{\nu i}\right)} \frac{\Delta N_{i j k}^{\nu(\bar{\nu})}}{\Delta x_{j} \Delta y_{k}}
$$

Events used in this analysis were triggered by the presence of a muon track. The data selection criteria for the cross section sample requires a good muon track, event containment, 
and minimum energies thresholds: $E_{\text {had }}>10 \mathrm{GeV}, E_{\mu}>15 \mathrm{GeV}$, and $E_{\nu}>30 \mathrm{GeV}$. To minimize the effects of the non-perturbative contributions, kinematic cuts of $Q^{2}>1 \mathrm{GeV}^{2}$ and $x<0.70$ are required.

The neutrino relative flux in energy bins is determined from a nearly independent sample at low hadronic energy $\left(E_{\text {had }}<20 \mathrm{GeV}\right)$. The absolute flux is obtained by normalizing the cross section to the world average value [3]. A detector simulation is used to account for acceptance and resolution effects. This simulation is based on a leading order QCD inspired cross section model [4] which is iteratively fit to the data. The final sample contains $8.6 \times 10^{5}$ neutrino $\left(\nu_{\mu}\right)$ and $2.4 \times 10^{5}$ anti-neutrino $\left(\bar{\nu}_{\mu}\right)$ events.

The following sources of experimental systematic uncertainties on the cross section measurement are considered: muon and hadron energy scales (which dominate) and smearing models, flux uncertainties, and the cross section model which is used to perform the acceptance corrections. The overall normalization uncertainty of the experiment is taken to be $2.1 \%$ from the normalization to the world average neutrino cross-section. The NuTeV data is presented together with a full point-to-point covariance matrix that provides the correlation coefficient between any two cross section data points [5].

\section{Comparison to other Neutrino Experiments}

The NuTeV differential cross section data are compared with measurements from other high statistics neautrino experiments, CCFR [6] and CDHSW [7] (comparison plots are published in [5] and presented in [1]). The three data sets are in reasonable agreement in both level and shape at $0.045<x<0.4$.

At $x>0.40$ CCFR's measurement for both $\nu$ and $\bar{\nu}$ cross sections are consistently below the $\mathrm{NuTeV}$ result over the entire energy range. Figure 1 shows the ratio of the CCFR and $\mathrm{NuTeV}$ differential cross sections as a function of $x$. The difference in the neutrino cross sections are $4 \pm 1 \%$ at $x=0.45$, $9 \pm 2 \%$ at $x=0.55$, and increases with $x$ up to $18 \pm 2 \%$ at $x=0.65$ (similarly for antineutrinos).

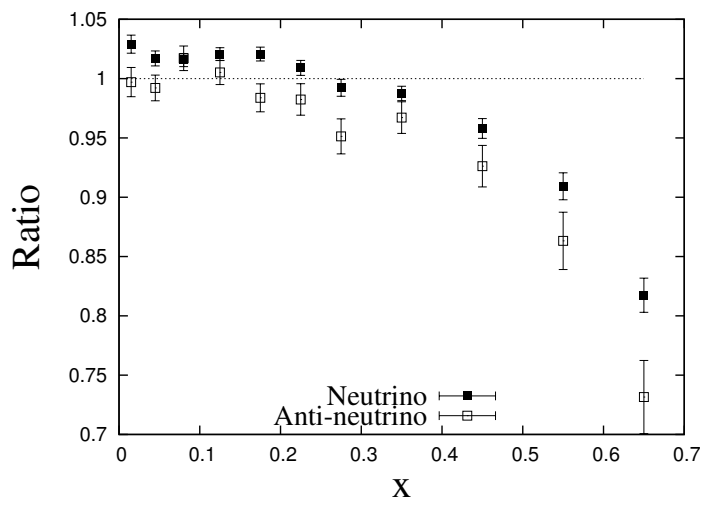

Figure 1: Ratio of CCFR to NuTeV cross sections as function of $x$ over all $E$ and $y$ for neutrinos (filled squares) and anti-neutrinos (open squares).

The largest single contribution to the discrepancy is due to an improved calibration of the magnetic field map of the toroid in $\mathrm{NuTeV}$ versus CCFR. NuTeV performed thorough calibrations of muon and hadron responses in the detector including mapping the response over the detector active area and measuring the energy scale over a wide range of energies [2]. This allowed for a precise determination of the the radial dependence of the magnetic field in the toroid. Employing the CCFR model the result is shifted to within 1.6 sigma agreement with CCFR at $x=0.65$. This accounts for $6 \%$ of the $18 \%$ difference at $x=0.65$. The field model differences can also be translated into an effective $0.8 \%$ difference in the muon energy scales by integrating the difference in the field models over the toroid. 
The cross section model contributes an additional $\sim 3 \%$ to the discrepancy seen at $\mathrm{x}=0.65$. Both experiments determine acceptance corrections using an iterated fit to the cross section data, this necessarily requires that the respective cross section models reflect the data differences.

Other smaller sources for the difference come from muon and hadron energy smearing models which results in a difference of $\sim 2 \%$ at $x=0.65$. All together these three contributions bring the two measurements within 1.2 sigma agreement in the high- $x$ region.

\section{Measurement of $\Lambda_{Q C D}$ from NLO QCD fits}

The $\Lambda_{Q C D}$ parameter is determined from NLO QCD fits to the structure functions: $x F_{3}\left(x, Q^{2}\right)$ only (non-singlet fit), and to both $F_{2}\left(x, Q^{2}\right)$ and $x F_{3}\left(x, Q^{2}\right)$ (combined fit). The non-singlet fit is independent of the gluon distribution. $x F_{3}\left(x, Q^{2}\right)$ is extracted from the 1-parameter fit to the $y$ dependence of the difference of $\nu$ and $\bar{\nu}$ differential cross sections (Eq. 1). The combined fit has an improved statistical precision for $\Lambda_{Q C D} . F_{2}\left(x, Q^{2}\right)$ and $x F_{3}\left(x, Q^{2}\right)$ are simultaneously extracted from the 2-parameter fits to the $\nu(\bar{\nu})$ cross sections. For this case input models for $\Delta x F_{3}$ (a NLO QCD model [10]) and $R_{L}$ (a fit to the world's measurements [11]) are needed.

Fixed-target DIS experiments are not sensitive to bottom quark excitation, therefore a $\Lambda_{Q C D}$ for the four active flavors is quoted. In order to minimize the nonperturbative QCD effects, only data points with $Q^{2}>5 \mathrm{GeV}^{2}$ and $W^{2}>10 \mathrm{GeV}^{2}$ are used in the NLO QCD fits. The evolution of parton densities starts at $Q_{0}^{2}=5$ $\mathrm{GeV}^{2}$, where $\Lambda_{Q C D}$ enters as a free parameter via integro-differential Dokshitzer-GribovLipatov-Altarelli-Parisi (DGLAP) evolution equations [12].

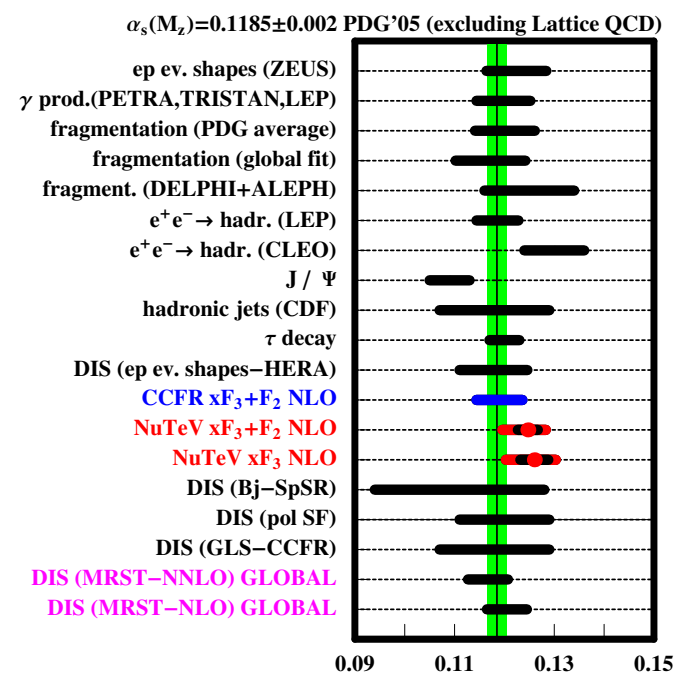

Figure 2: $\mathrm{NuTeV} \alpha_{S}\left(M_{Z^{0}}\right)$ in red compared to other experimental measurements [8]. The errors include both experimental and theoretical uncertainties.

Neutrino Scattering is sensitive to heavy quark production, therefore the QCD fits are performed using Aivazis-Collins-Olness-Tung (ACOT) model [13] that takes into account heavy quark production [14]. The following results for $\Lambda_{Q C D}^{n_{f}=4}$ with its corresponding $\alpha_{S}\left(M_{Z}\right)$ are obtained from non-singlet and combined fits, respectively:

$$
\begin{aligned}
& \Lambda_{A C O T}^{N L O,(n=4)}=488 \pm 59(s t a+s y s)_{-113}^{+74}(t h) ; \quad \alpha_{S}\left(M_{Z}\right)=0.1260 \pm 0.0028_{-0.0050}^{+0.0034} ; \\
& \Lambda_{A C O T}^{N L O,(n=4)}=458 \pm 41(s t a+s y s)_{-87}^{+61}(t h) ; \quad \alpha_{S}\left(M_{Z}\right)=0.1247 \pm 0.0020_{-0.0047}^{+0.0030} \text {. }
\end{aligned}
$$

The first error corresponds to total experimental uncertainties (statistical and systematic). The second error is the estimated theoretical uncertainty from the factorization scale dependence (dominant uncertainty). The systematic uncertainties associated with quark masses are found to be small [15]. The experimental systematic uncertainties are propagated from 
the extracted differential cross section described in Section 2. The fits are performed using the full covariance error matrix. The largest experimental systematic errors are due to the energy scales and the smearing models, which are of the order of statistical precision.

Figure 2 shows the comparison of the NuTeV measurements of $\alpha_{S}\left(M_{Z}\right)$ to those of other experiments [8]. The band denotes the world weighted average, as quoted by [8]. The NuTeV results situate above the world average, but agree within errors.

\section{Conclusions}

The most precise measurement to date of neutrino and anti-neutrino iron differential cross section in the energy range $E_{\nu}>30 \mathrm{GeV}$, published in [5], has been presented [1]. The systematic uncertainty from the energy scale has been reduced over previous neutrino measurements by about a factor of two. For the first time a full covariance matrix is provided. This data can be used in global fits to constrain the parton distribution functions which are essential to the collider community in calculating cross-sections for particle production and constraining new physics.

A new measurement of $\Lambda_{Q C D}$ from Next-to-Leading Order QCD fits to the $Q^{2}$ dependence of neutrino-iron structure functions has been summarized [1] (more details are found in [15]). This is the first measurement of $\Lambda_{Q C D}$ employing a NLO theoretical model which fully accounts for heavy quark production. The NuTeV measurements of $\Lambda_{Q C D}$ from nonsinglet and combined NLO QCD fits, translated into $\alpha_{S}\left(M_{Z}\right)=0.1247 \pm 0.0020_{-0.0047}^{+0.0030}$ and $\alpha_{S}\left(M_{Z}\right)=0.1260 \pm 0.0028_{-0.0050}^{+0.0034}$, respectively, agree within errors with the world average value. This measurement is one of the most precise determination of $\alpha_{S}$ to date.

\section{Bibliography}

\section{References}

[1] Slides: http: //indico. cern. ch/contributionDisplay $\cdot$ py? contribId=282\&sessionId=14\&conf Id=9499

[2] D. A. Harris et al., Nucl. Instrum. Methods A 447 (2000) 377.

[3] W. Seligman, Ph. D. Thesis, Columbia University, Nevis 292 (1997).

[4] A. J. Buras and K. L. F. Gaemers, Nucl. Phys. B 132 (1978) 2109.

[5] M. Tzanov et al. (NuTeV Collaboration), Phys. Rev. D74 012008 (2006);

[6] U. K. Yang, Ph. D. Thesis, University of Rochester, (2001).

[7] P. Berge et al., Z. Phys.C (1991) 187.

[8] Particle Data Group Listing (2005).

[9] Bardin, D. Y. and Dokuchaeva, JINR-E2-86-260 (1986).

[10] R. S. Thorne and R. G. Roberts, Phys. Lett. B 421 (1998) 303;

A. D. Martin et al. Eur. Phys. J. C 18 (2000) 117.

[11] L. W. Whitlow et al., Phys. Lett. B 250 (1990) 193.

[12] Yu. L. Dokshitzer, Sov.Phys.JETP46 (1977);

[13] M. A. G. Aivazis, J. C. Collins, F. I. Olness and W. K. Tung Phys. Rev. D 50, (1994) 3102.

[14] F. I. Olness, J. F. Owens, private communication.

[15] V. Radescu, Ph. D. Thesis, University of Pittsburgh, (2006). 